\title{
Correction to: Efficacy and Safety Results from a Phase 2, Randomized, Double Blind Study of Enzalutamide Versus Placebo in Advanced Hepatocellular Carcinoma
}

\author{
Baek-Yeol Ryoo ${ }^{1}$. Daniel H. Palmer ${ }^{2}$. Sook Ryun Park ${ }^{1}$. Lorenza Rimassa ${ }^{3,4} \cdot$ Debashis Sarker $^{5} \cdot$ Bruno Daniele $^{6,7}$. \\ Joyce Steinberg ${ }^{8} \cdot$ Beatriz López $^{9,10} \cdot$ Ho Yeong Lim ${ }^{11}$
}

Published online: 27 February 2022

(c) Springer Nature Switzerland AG 2022

\section{Correction to: \\ Clinical Drug Investigation (2021) 41:795-808 \\ https://doi.org/10.1007/s40261-021-01063-0}

The original version of this article unfortunately contained a mistake. The given name and family name of the fifth author was incorrectly tagged in the xml data, therefore it is listed wrongly as "Debashis Sarker" in Pubmed. The correct given name is Debashis and family name is Sarker.
The original article can be found online at https://doi.org/10.1007/ s40261-021-01063-0.

Baek-Yeol Ryoo

ryooby@amc.seoul.kr

1 Department of Oncology, Asan Medical Center, University of Ulsan College of Medicine, Seoul, South Korea

2 Liverpool CR UK/NIHR Experimental Cancer Medicine Centre, and The Clatterbridge Cancer Centre, Liverpool, UK

3 Department of Biomedical Sciences, Humanitas University, Pieve Emanuele, Milan, Italy

4 Medical Oncology and Hematology Unit, Humanitas Cancer Center, IRCCS Humanitas Research Hospital, Rozzano, Milan, Italy

5 School of Cancer and Pharmaceutical Sciences, King's College London, London, UK
6 Department of Oncology, G. Rummo Hospital, Benevento, Italy

7 Present Address: Oncology Unit, Ospedale del Mare, Naples, Italy

8 Astellas Pharma Inc., Northbrook, IL, USA

9 Data Science, Astellas Pharma Inc., Leiden, The Netherlands

10 Present Address: Quantitative Sciences, Janssen R\&D, Janssen Pharmaceutical Companies of Johnson \& Johnson, Leiden, The Netherlands

11 Division of Hematology-Oncology, Department of Medicine, Samsung Medical Center, Sungkyunkwan University School of Medicine, Seoul, South Korea 